\title{
The NIR transmission spectrum of Jupiter from the observation of a Ganymede's eclipse
}

\author{
P. Montañés-Rodríguez ${ }^{1,2, a}$, E. Pallée ${ }^{1,2}$, B. González-Merino ${ }^{1,2}$, M. López-Puertas ${ }^{3}$, and E. \\ García-Melendo ${ }^{4}$ \\ 1 Instituto de Astrofísica de Canarias, C/Vía Láctea s/n, E-38200 La Laguna, Spain \\ 2 Departamento de Astrofísica, Universidad de La Laguna, Av., Astrofísico Francisco Sánchez, s/n, \\ E-38206 La Laguna, Spain \\ 3 Instituto de Astrofísica de Andalucía (CSIC), Glorieta de la Astronomía s/n, E-18080 Granada, \\ Spain \\ 4 Departamento de Física Aplicada I, E.T.S. Ingeniería, Universidad del País Vasco, 9 Alameda Urquijo \\ s/n, 48013 Bilbao, Spain.
}

\begin{abstract}
Here, we report the NIR transmission spectrum of Jupiter, with high signalto-noise ratio, as if it were a transiting planet. Our technique was to observe Ganymede, when crossing Jupiter's shadow. During the eclipse, the spectral features of the Jovian atmosphere are imprinted in the sunlight that, after passing through Jupiter's planetary limb, is reflected from Ganymede towards the Earth. The ratio spectrum of Ganymede before and during the eclipse removes the spectral features of the Sun, the local telluric atmosphere on top of the telescopes, and the spectral albedo of Ganymede. Ganymede is a practically atmosphere-less body and do not suffer any significant weather variability.
\end{abstract}

\section{Introduction}

Over the past two decades, more than 1800 exoplanets have been discovered [4], approximately 65\% of which are transiting. During the transit of a planet in front of a star the stellar flux is partially blocked, but a very small fraction of the stellar flux passes through the thin planetary atmosphere. Transmission spectroscopy has allowed the detection of many atmospheric components in exoplanets $[1-3,6]$. These observations, however, push the detection capabilities of space and ground-based observatories to the limit and the results are often a source of discrepancies [5,7], which need to be addressed. Observing planetary transits in our own Solar System can provide crucial information for exoplanet characterization.

\section{Observations}

We observed an eclipse of Ganymede on 6/10/2012 with LIRIS [8], which is a near-IR spectrograph in use at the Cassegrain focus of the 4.2-m William Herschel Telescope (WHT). We measured the evolution of the spectrum of Ganymede, which initially corresponded to direct sunlight, followed by the penumbra and the first minutes of the umbra phases, using the zJ grism (covering the 0.887 to 1.531 $\mu m$ spectral range at $\mathrm{R}=700$ ). When in the deep umbra, Ganymede was undetectable, but we continued to make exposures on target, and we switched to the hk grism (covering 1.388-2.419 $\mu \mathrm{m}$, at $\mathrm{R}=700$ ). With the later configuration we observed the last minutes of umbra (when the satellite became bright enough again), the penumbra, and out-of-the-eclipse. We used a 1" wide and 4.2' long slit. A total of

a e-mail: pmr@iac.es

This is an Open Access article distributed under the terms of the Creative Commons Attribution License 4.0, which permits unrestricted use, distribution, and reproduction in any medium, provided the original work is properly cited. 


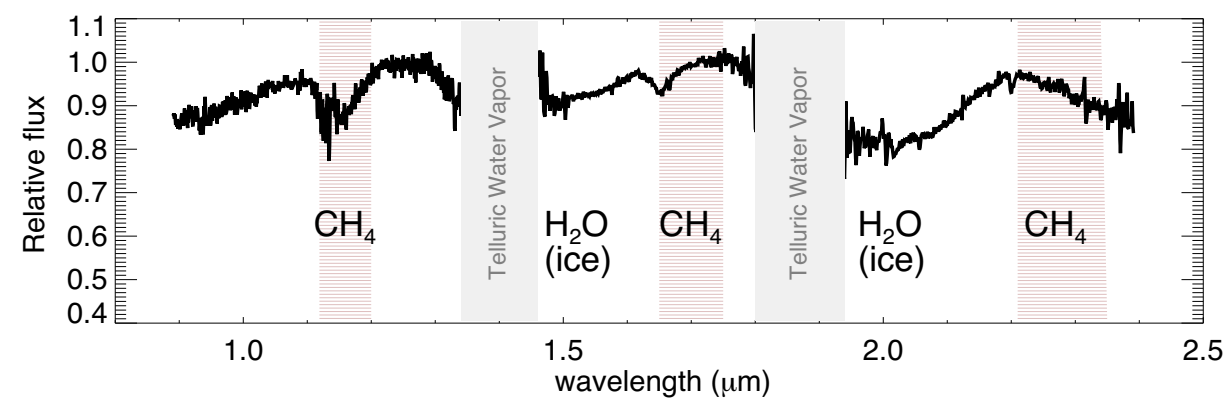

Fig. 1. The transmission spectrum of Jupiter as measured with LIRIS at the WHT telescope during the umbral phase of the eclipse. Gray shaded regions mark the deeply absorbed telluric bands of $\mathrm{H}_{2} \mathrm{O}$, which cannot be observed from the ground. The most important molecular features in the spectrum are labelled. The comparison with recent models developed during the last months of 2014 show the fingerprints of gaseous $\mathrm{CH}_{4}$ and crystallized $\mathrm{H}_{2} \mathrm{O}$ ices, which were still not evident to us during the symposium.

1524 spectra were collected between 03:00 and 06:40 UT covering the entire event. The airmass variation during the observations was very small (1.16 to 1.07), and decreased with time. This means that the bright spectra are taken at higher airmass than the (pen-)umbra, and thus telluric contamination is observed in absorption. The apparent magnitude of Ganymede is $\mathrm{V}=4.6$, and it decreases several magnitudes during the umbra phase. Therefore, local atmospheric conditions and the well centering of the satellite image on the slit during penumbra and umbra were critical aspects of the acquisition. We attempted, unsuccessfully due to weather conditions, to observe a previous eclipse on 19/02/2012.

The retrieved Jupiter transmission spectrum shows strong absorption features from $\mathrm{CH}_{4}$. More interestingly, a spectral signature appears, which we tentatively attribute to a stratospheric layer of $\mathrm{H}_{2} \mathrm{O}$ ice clouds. We plan to conduct a detailed comparison with radiative transfer models to confirm it. $\mathrm{CO}_{2}$ and $\mathrm{NH}_{3}$ remain undetected, indicating that these components are below the average altitudes sampled with our method. Our results are relevant for the modelling and interpretation of giant transiting exoplanets.

Based on observations made with the William Herschel Telescope at the Observatorio del Roque de los Muchachos, La Palma. This work is partly financed by the Spanish MINECO through projects SEV-2011-0187 (2011 Severo Ochoa Program), AYA2012-39612-C03-02, AYA2010-21308-C03-02, AYA2011-30147-C03-03, AYA201236666, and AYA2011-23552. The authors wish to thank to the ING support astronomers team.

\section{References}

1. Huitson, C. M., Sing, D. K. et al., MNRAS 434, (2013) 3252-3274

2. Tinetti, G., Deroo, P. et al., ApJ 712, (2010) L139-L142

3. Bellucci, G. et al., Advances in Space Research 34, 8, (2004) 1640-1646

4. Borucki, et al., Science 327, (2010) 977

5. Ehrenreich, G., et al., A\&A 448, (2006) 379-393

6. Sing, D. K., et al., MNRAS 416, (2011) 1443-1455

7. Gibson et al., MNRAS 411, (2011) 2199

8. Manchado, A., M. Barreto, J. Acosta-Pulido, et al, SPIE 5492, (2004) 1094-1104 